\title{
A Trajectory Compensation Model for Roll Hemming Applications
}

\author{
Eduardo Esquivel González, ${ }^{1,}$ - José Pablo Rodríguez Arzate ${ }^{2}$ - Giuseppe Carbone ${ }^{3}$ \\ - Marco Ceccarelli ${ }^{3}$ - Juan Carlos Jáuregui ${ }^{1}$ \\ ${ }^{1}$ Autonomous University of Querétaro, Faculty of Engineering, México \\ ${ }^{2}$ Technological Institute of Celaya, Department of Mechatronics, México \\ ${ }^{3}$ University of Cassino, Laboratory of Robotics and Mechatronics, Italy
}

This paper presents a trajectory compensation model to correct the deviation in the roll hemming applications. First, the main defects and problems of roll hemming technology are established. A trajectory compensation proposal is analyzed as well as the kinematic and stiffness model of the robot and the material deformation model. The implementation of the model on an industrial robot is tested and simulated. Consequently, the viability of the model is discussed and compared with other works.

Keywords: roll hemming, wrinkling, tool center point, stiffness, trajectory
Highlights
- An offline compensation strategy is implemented for roll hemming.
- The compensation strategy relies on the deviation due to the robot.
- The compensation strategy relies on the deviation due to the panel.
- The results show the new trajectory within the main parameters of the process.

\section{O INTRODUCTION}

Robots in automotive applications offer low-cost solutions for most of the manufacturing processes, opening new possibilities ranging from simple tasks, like pick and place, painting and sealing, to more complex tasks, like milling or welding [1]. This allows replacing the computer-navigated control (CNC) machines and stamping machines by using robotic manipulators in metal forming processes. They are also applicable for new processes like roll hemming, commonly used for doors, hoods and deck lids of the automotive industry. In the process, a serial robot moves a roller through the pre-hemming steps over the contour in order to attach the exterior panel to the interior panel of a door [2].

The roll hemming offers flexibility but different defects may appear in the final panel's shape. A common visible defect is the formation of waves on the flange, called wrinkles, related to the velocity and force of the roller. The origin of such defects relies on the capacity of the roller to deform the panel depending on the robot's pose. For example, an extended configuration pose demands higher torque and applies lower force than a constricted one that requires less torque and applies higher force. The stiffness of a robot $(1 \mathrm{~N} / \mu \mathrm{m})$ is lower compared to a CNC machine $(50 \mathrm{~N} / \mu \mathrm{m})$ [3], decreasing its capacity to follow a designated trajectory under external forces. As consequence, the final quality of the panel may vary along the trajectory. Most of the works of roll hemming process [4] are related to the finite element analysis and the prediction of the deformation patterns of the panel and few works [5] are focused on the dynamic performance of the robot. Accordingly, this paper proposes a compensation strategy for the roll hemming process based on the variable stiffness of the robot to minimize the tool deviation along the trajectory.

This paper proposes an offline compensation model for roll hemming with emphasis on determining the stiffness of the robot. A simulation of the process has been developed and the experimental tests show the error's results. If this compensation strategy is implemented, then a more accurate solution is achieved and a better quality of the product can be guaranteed.

This paper is structured in three main sections. The methods section establishes the trajectory compensation proposal and the analysis of each element concluding with the integration of the trajectory compensation model. The experimental section describes how the stiffness values were determined and how the forces and speed of the process were related to the wrinkling defect of the panel. Finally, the results section shows the values obtained from the computation of the algorithm and the deviation due to the error of the trajectory. 


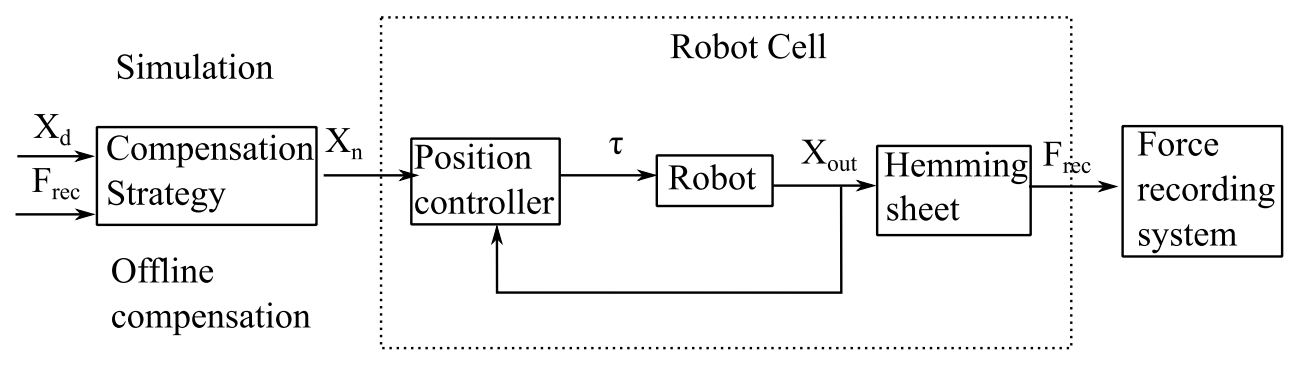

Fig. 1. An offline compensation strategy based on the variable force of the process

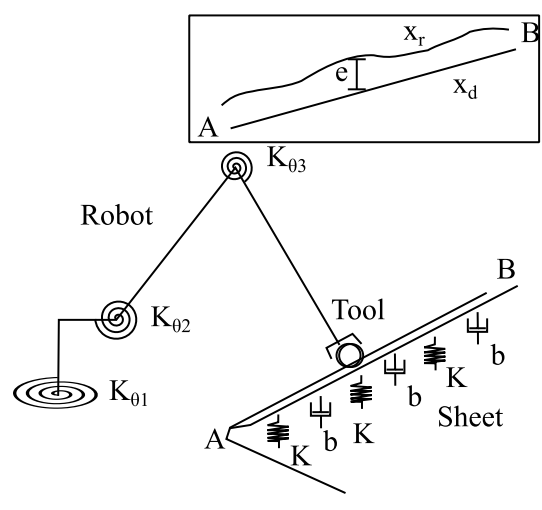

Fig. 2. The model of the roll hemming process

\section{METHODS}

\subsection{A Trajectory Compensation Proposal}

An offline compensation approach is defined in this paper. The Fig. 1 shows a trajectory compensation proposal related to the robot cell and the roll hemming process, where the desired position $X_{d}$ and the recorded force $F_{\text {rec }}$ are the inputs of the model. Once computed the new trajectory $X_{n}$, the position controller commands the robot toward the hemming process. The $X_{\text {out }}$ represents the location's feedback.

The Fig. 2 shows the scheme of the process as the robot deforms the flange. The first three joints of the robot are modeled as torsional springs and the last three joints are used for orientation having a lower impact on the elasticity behavior. The sheet is modeled as linear spring with a damping effect. The tool center point (TCP) moves from position A to position B along its path but the material stiffness and the elasticity of the robot affect the desired trajectory $X_{d}$ producing an error $e$ in comparison with the real trajectory $X_{r}$.

A representative equation of the compensation strategy is

$$
\begin{aligned}
& X_{n}=X_{d}+e, \\
& X_{n}=X_{d}+\Delta_{\text {robot }}+\Delta_{\text {sheet }},
\end{aligned}
$$

where $e$ is the term of the error or deviation.

\subsubsection{The Deviation Due to the Robot}

This section describes the mathematical model to determine the deflection of the robot $\Delta_{\text {robot }}$. The deflection vector has three terms for position and three for orientation due to the configuration and the stiffness of the robot. The general deflection equations are built from the stiffness in the Cartesian and joint spaces as

$$
F=K_{x} \delta_{x}, \tau=K_{\theta} \delta_{\theta},
$$

where the vector $F$ is the $6 \times 1$ vector of external forces and torques applied to the tool, the matrix $K_{x}$ is the $6 \times 6$ Cartesian stiffness matrix, the vector $\delta_{x}$ is the $6 \times 1$ vector of linear and angular displacements, the vector $\tau$ is the $6 \times 1$ vector of joint torques, the matrix $K_{\theta}$ is the $6 \times 6$ joint stiffness matrix and the vector $\delta_{\theta}$ is the $6 \times 1$ vector of joint displacements. Both displacements are related through the Jacobian relation

$$
\delta_{x}=J(\Theta) \delta_{\theta},
$$

where the variable $J(\Theta)$ represents the $6 \times 6$ Jacobian matrix. By inserting this equation in Eq. (2) a new equation is obtained

$$
\tau=K_{\theta} J^{-1}(\Theta) \delta_{x}
$$

this equation relates the two different spaces by the Jacobian matrix. Naming the principle of virtual work

$$
F^{T} \delta_{x}=\tau^{T} \delta_{\theta}
$$

and combining this relation with Eq. (3) another equation is formed

$$
\tau=J^{T}(\Theta) F
$$

which is similar to Eq. (3). Again, a substitution in Eq. (4) results in a new statement

$$
J^{T}(\Theta) F=K_{\theta} J^{-1}(\Theta) \delta_{x} .
$$


Table 1. Denavit-Hartenberg parameters for the robot Fanuc 200IC

\begin{tabular}{ccccc}
$i$ & $\alpha_{i-1}$ & $a_{i-1}$ & $d_{i}$ & $\theta_{i}$ \\
\hline \hline 1 & 0 & 0 & 0 & $\theta_{1}$ \\
\hline 2 & $-\frac{\pi}{2}$ & $a_{1}$ & 0 & $\theta_{2}-\frac{\pi}{2}$ \\
\hline 3 & $\pi$ & $a_{2}$ & 0 & $\theta_{3}$ \\
\hline 4 & $-\frac{\pi}{2}$ & $a_{3}$ & $-d_{4}$ & $\theta_{4}$ \\
\hline 5 & $\frac{\pi}{2}$ & 0 & 0 & $\theta_{5}$ \\
\hline 6 & $-\frac{\pi}{2}$ & 0 & 0 & $\theta_{6}$ \\
\hline $\mathrm{T}$ & 0 & 0 & $-d_{t}$ & 0 \\
\hline
\end{tabular}

This last equation relates the force $F$ and the displacement $\delta_{x}$ of the Cartesian space with the joint stiffness matrix. Expressing in explicit form

$$
\Delta_{\text {robot }}=\delta_{x}=K_{\theta}^{-1} J(\Theta) J^{T}(\Theta) F^{W} .
$$

Observing this equation it is noted that the tool displacement depend on the force, the jacobian matrix and the joint stiffness matrix. The force and displacement are required to be expressed in the global reference frame. A general view of the last equation shows a similarity to Eq. (2) where forces and displacements are related through the Cartesian stiffness matrix.

\subsubsection{The Kinematic Model of the Robot}

The Fig. 3 shows the scheme of the robot with the reference frames attached to each joint and with 6 degrees of freedom. All the joints were considered as flexible and all the links as rigid bodies, the Table 1 shows the Denavit-Hartenberg parameters established for this robot, being $a_{1}=75 \mathrm{~mm}, a_{2}=300 \mathrm{~mm}, a_{3}=$ $75 \mathrm{~mm}, d_{1}=330 \mathrm{~mm}, d_{4}=320 \mathrm{~mm}$, and the distance to the TCP $d_{t}=80 \mathrm{~mm}+120 \mathrm{~mm}$, this parameters describe the kinematic behavior of the robot. The Jacobian matrix is proposed in order to relate the Cartesian displacement and the joint displacement as

$$
J(\Theta)=\left(\begin{array}{lll}
J(\Theta)_{1} & J(\Theta)_{2} & J(\Theta)_{3}
\end{array}\right)^{T},
$$

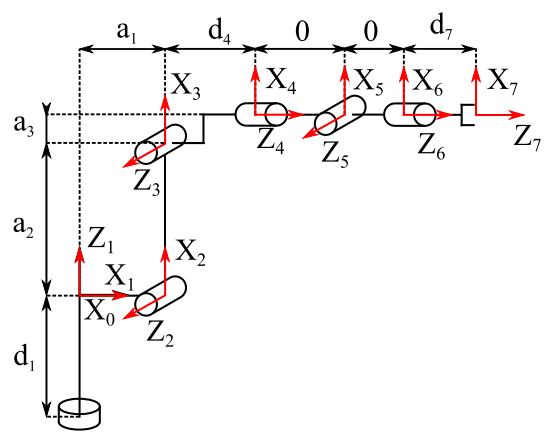

Fig. 3. The scheme of the robot with the reference frames where each one of the terms is expressed as

$$
J(\Theta)_{1}=\left(\begin{array}{c}
-\sin \left(\theta_{2}+\theta_{3}\right) d_{1} \\
\sin \left(\theta_{1}+\theta_{2}\right)-\cos \left(\theta_{2}+\theta_{3}\right) d_{4}+\sin \theta_{2} a_{2}-a_{1} \\
\cos \left(\theta_{2}+\theta_{3}\right) d_{1}
\end{array}\right) .
$$

The second column corresponds to

$$
J(\Theta)_{2}=\left(\begin{array}{c}
\sin \theta_{3} a_{2}+d_{4} \\
0 \\
-\cos \theta_{3} a_{2}-a_{3}
\end{array}\right),
$$

and the third column corresponds to

$$
J(\Theta)_{3}=\left(\begin{array}{c}
d_{4} \\
0 \\
-a_{3}
\end{array}\right) .
$$

This is the jacobian matrix considered for the deviation due to the robot.

\subsubsection{A Method to Compute the Stiffness/Compliance of the Robot}

This paper proposes to compute the joint stiffness matrix $K_{\theta}$ as a function of the vector of external forces and torques and the vector of linear and angular displacement expressed as

$$
K_{\theta}=f\left(F, \delta_{x}\right)
$$

In order to obtain the last expression, the Eq. (8) is reordered in explicit form for the compliance vector as

$$
\delta_{x}=\left(\begin{array}{c}
\frac{1}{K_{\theta 1}}\left[\Sigma_{j=1}^{6}\left[J_{1 j}\left(\Sigma_{i=1}^{6} j_{i j} F_{i}\right)\right]\right] \\
\frac{1}{K_{\theta 2}}\left[\Sigma_{j=1}^{6}\left[J_{2 j}\left(\Sigma_{i=1}^{6} j_{i j} F_{i}\right)\right]\right] \\
\frac{1}{K_{\theta 3}}\left[\Sigma_{j=1}^{6}\left[J_{3 j}\left(\Sigma_{i=1}^{6} j_{i j} F_{i}\right)\right]\right] \\
\frac{1}{K_{4}}\left[\Sigma_{j=1}^{6}\left[J_{4 j}\left(\Sigma_{i=1}^{6} j_{i j} F_{i}\right)\right]\right] \\
\frac{1}{K_{\theta 5}}\left[\Sigma_{j=1}^{6}\left[J_{5 j}\left(\Sigma_{i=1}^{6} j_{i j} F_{i}\right)\right]\right] \\
\frac{1}{K_{\theta 6}}\left[\Sigma_{j=1}^{6}\left[J_{6 j}\left(\Sigma_{i=1}^{6} j_{i j} F_{i}\right)\right]\right]
\end{array}\right),
$$

where the compliance matrix $K_{\theta}^{-1}$ has been integrated in the resultant vector of the displacement and substituted for the vector of compliance $C$

$$
C=\left[\frac{1}{K_{\theta 1}} \frac{1}{K_{\theta 2}} \frac{1}{K_{\theta 3}} \frac{1}{K_{\theta 4}} \frac{1}{K_{\theta 5}} \frac{1}{K_{\theta 6}}\right],
$$

it is possible to present the Eq. (14) in the form

$$
\delta_{x}=A C,
$$

where $A$ is formed by the Jacobian and force terms. This equation is expressed as a linear matrix equation $A x=b$ where the unknown values are those of the vector of compliance $C$. If $A C=\delta_{x}$ has no solution 


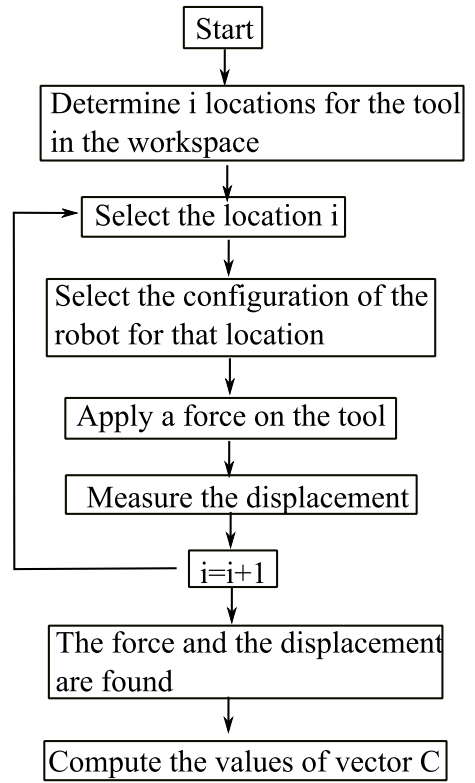

Fig. 4. The flow chart to find the values of the compliance

by finding $A^{-1}$, then we can find the minimum error $e=A C-\delta_{x}$ by the least square solution

$$
C_{0}=\left(A^{T} A\right)^{-1} A^{T} \delta_{x}
$$

where the vector of compliance $C_{0}$ relates every joint deflection to a specific torque. The precision of the compliance vector is related to the measurement of the forces and displacements of the tool. The Fig. 4 illustrates the procedure to determine the values of the compliance where the first steps is to determine the location $i$ of the work-space to test the values of displacements and forces, the second step is to select a specific location for which the robot takes the specific configuration to reach that location, once in that configuration a specific force is applied on the tool and the displacements are measured, the procedure is repeated until covering the work-space and finally the Eq. (17) computes the values in a numerical software.

\subsubsection{The Deviation Due to the Panel}

The deviation due to the panel can be computed as

$$
\Delta_{\text {sheet }}=\Delta_{e p}+\Delta_{\text {wrinkling }},
$$

where the total deflection of the sheet's flange $\Delta_{\text {sheet }}$ is the sum of the elastic and plastic deformation $\Delta_{e p}$ and the deformation due to the wrinkling defect $\Delta_{\text {wrinkling }}$ produced from the waves of the flange. This model gives a contribution to the common finite element models presented in the roll hemming overview. The Fig. 5 shows how the external force $F$, a scalar

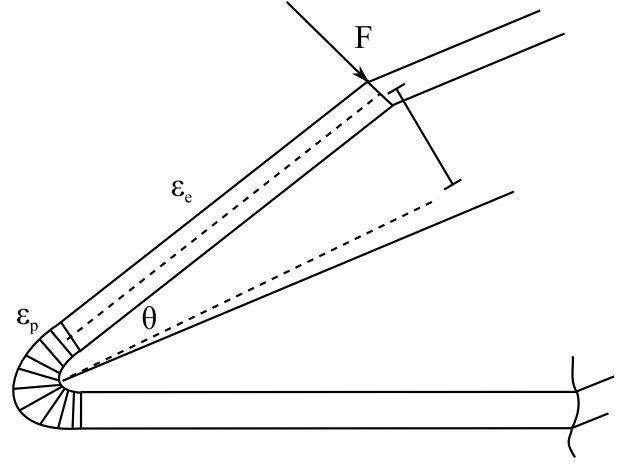

Fig. 5. The material deformation scheme

value for the panel, generates the plastic $\varepsilon_{p}$ and elastic $\varepsilon_{e}$ deformations as the flange inclines the angular position $\theta$. This scheme can be modeled in the form $\varepsilon=\varepsilon_{e}+\varepsilon_{p}$ where the total deformation of the sheet $\varepsilon$ is the sum of the elastic $\varepsilon_{e}$ and the plastic $\varepsilon_{p}$ deformation. The elastic deformation $\varepsilon_{e}$ may be computed by considering the flange as a beam under bending load at the end for a specific section of the material. To model this deflection two relations are considered

$$
1 / R=\frac{M}{E I_{z}}, \quad \sigma=\frac{M y}{I_{z}},
$$

where $R$ is the radius of curvature, $M$ is the bending moment, $E$ is the Young's modulus, $I_{z}$ is the second moment of area, $\sigma$ is the stress of the beam and $y$ is the distance from the neutral middle line on the beam towards the external fibers. The use of these two equations results in the deflection function

$$
\delta_{e}=\frac{F x^{2}(x-3 l)}{6 E I_{z}},
$$

where $F$ is the load at the end of the flange, $x$ is the distance at any given section of the flange, and $l$ is the total length of the flange. If it is considered the distance $x=l$ for deflection, then the Eq. (20) becomes

$$
\delta_{e}=-\frac{F l^{3}}{3 E I_{z}}=\frac{Y l^{2}}{3 E h}
$$

which states the deflection for the elastic part of the material at the end of a beam, where $Y$ is the yield stress and $h$ is the total height of the cross section from the neutral line to the external fiber.

Chakrabarty established that the longitudinal strain in a elastic beam is $\varepsilon_{x}=y / R$ and the transverse strains are $\varepsilon_{y}=\varepsilon_{z}=-v y / R$, where $v$ is the Poisson's ratio [6]. By using these relations the deflection $v$ on 


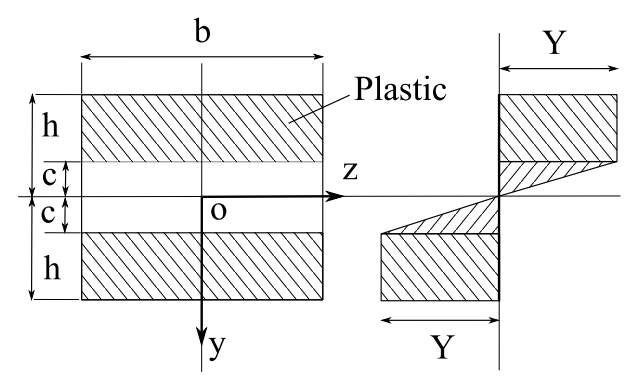

Fig. 6. The plastic deformation on the rectangular cross section

the $y$ axis is established as

$$
v=\frac{x^{2}+v\left(y^{2}-z^{2}\right)}{2 R} .
$$

The Fig. 6 shows the rectangular cross section during plastic deformation assuming an elastic, perfectly plastic behavior, where $b$ is the width of the section. The bending moment $M_{e}$ and the radius of curvature $R_{e}$ at the elastic limit are established in view of Eq. (19) using an area moment of inertia $I_{z}=\frac{2 b h^{3}}{3}$ at the initial yielding stress when $y=h$

$$
M_{e}=\frac{2 b h^{2} Y}{3}, \quad R_{e}=\frac{E h}{Y}, \quad R=\frac{E c}{Y},
$$

where $R$ is the radius of curvature at any stage during the elastic/plastic bending. Then according to the material strain-hardening

$$
\frac{\sigma}{Y}=\left(\frac{E \varepsilon}{Y}\right)^{n},
$$

where $0 \leq n \leq 1$, $n$ being the strain hardening exponent, and with the condition $\varepsilon \geq Y / E$. By considering that $\varepsilon=y / R$

$$
\sigma=\left\{\begin{array}{ll}
Y\left(\frac{y}{c}\right), & 0 \leq y \leq c \\
Y\left(\frac{y}{c}\right)^{n}, & c \leq y \leq h
\end{array} .\right.
$$

The bending moment at any stage of the plastic deformation is

$$
M=2 b \int_{0}^{h} \sigma y d y .
$$

By substituting Eq. (25) into Eq. (26) and integrating we obtain

$$
\frac{M}{M_{e}}=\frac{1}{2+n}\left[3\left(\frac{R_{e}}{R}\right)^{n}-(1-n)\left(\frac{R}{R_{e}}\right)^{2}\right] .
$$

The above equation applies for a non hardening material when $n=0$ obtaining

$$
\frac{R_{e}}{R}=\left\{\begin{array}{ll}
\frac{M}{M_{e}}, & M \leq M_{e} \\
\left(3-2 \frac{M}{M_{e}}\right)^{-1 / 2}, & M \geq M_{e}
\end{array} .\right.
$$

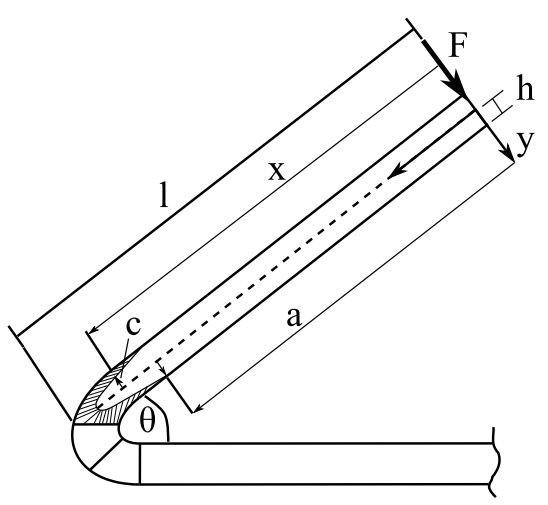

Fig. 7. The plastic deformation model.

Considering the second partial derivative of Eq. (22) respect to $x$ we obtain

$$
\frac{1}{R}=-\frac{\partial^{2} v}{\partial x^{2}}
$$

The Fig. 7 illustrates a frontal view of the flange as a cantiliver beam with a terminal load, where $l$ is the total length of the flange and $\theta$ is the inclination angle. This assumption involves the following relations

$$
\frac{F_{e}}{F}=\frac{a}{l}, \quad \frac{M}{M_{e}}=-\frac{x}{l},
$$

where $F_{e}$ is the load at the elastic/plastic boundary, $a$ is the distance to the elastic/plastic boundary and $M_{e}$ is the bending moment at the elastic/plastic boundary. By inserting Eq. (29) and Eq. (30) into the Eq. (28) we obtain

$$
R_{e} \frac{\partial^{2} v}{\partial x^{2}}=\left\{\begin{array}{ll}
\left(3-2 \frac{x}{a}\right)^{-1 / 2}, & x \geq a \\
\frac{x}{a}, & x \leq a
\end{array} .\right.
$$

for the case where $a \leq x \leq l$, we integrate the equation, knowing that $\partial v / \partial x$ vanishes when $x=l$ as a boundary condition, resulting

$$
\frac{\partial v}{\partial x}=-\frac{a}{R_{e}}\left[\sqrt{3-2 \frac{x}{a}}-\sqrt{3-2 \frac{l}{a}}\right] .
$$

The equation is integrated again applying the same boundary condition, the deflection $v$ vanishes at $x=l$ obtaining

$$
v=\frac{a^{2}}{3 R_{e}}\left[\left(3-\frac{2 x}{a}\right)^{3 / 2}-\left(3+\frac{l-3 x}{a}\right) \sqrt{3-\frac{2 l}{a}}\right] .
$$

The Eq. (31) of limits $0 \leq x \leq a$ is integrated considering that $\partial v / \partial x$ is continuous across $x=a$, and we obtained

$$
\frac{\partial v}{\partial x}=-\frac{a}{R_{e}}\left[\frac{1}{2}\left(3-\frac{x^{2}}{a^{2}}\right)-\sqrt{3-\frac{2 l}{a}}\right] .
$$


Integrating again this equation and considering that $v$ is continuous across $x=a$ we obtain

$$
v=\frac{a^{2}}{3 R_{e}}\left[5-\frac{x}{2 a}\left(9-\frac{x^{2}}{a^{2}}\right)-\left(3+\frac{l-3 x}{a}\right) \sqrt{3-\frac{2 l}{a}}\right] .
$$

The last equation represents the deflection at any distance $x$ of the flange due to the plastic conditions. Knowing that

$$
\delta_{e}=\frac{Y l^{2}}{3 E h}=\frac{l^{2}}{3 R_{e}},
$$

and multiplying the left side of the Eq. (35) for the unity $l^{2} / l^{2}$, then the term $\delta_{e}$ appears in the equation.

$$
v=\frac{a^{2}}{l^{2}}\left[5-\frac{x}{2 a}\left(9-\frac{x^{2}}{a^{2}}\right)-\left(3+\frac{l-3 x}{a}\right) \sqrt{3-\frac{2 l}{a}}\right] \delta_{e} .
$$

If we apply $x=0$ to the corresponding equation and considering the load (at the end of the deflection) then

$$
\Delta_{e p}=v=\left(\frac{F_{e}}{F}\right)^{2}\left[5-\left(3+\frac{F}{F_{e}}\right) \sqrt{3-\frac{2 F}{F_{e}}}\right] \delta_{e} .
$$

This equation contains the elastic/plastic deflection. By substituting the Eq. (21) into the last one, a new expression is obtained

$$
\Delta_{e p}=-\left(\frac{F_{e}}{F}\right)^{2}\left[5-\left(3+\frac{F}{F_{e}}\right) \sqrt{\left.3-\frac{2 F}{F_{e}}\right] \frac{F l^{3}}{3 E I}} .\right.
$$

The deflection is presented respect to the $z$ axis of the tool reference frame. This relation expresses the first term of the Eq. (18).

A form to represent the wrinkling deviation $\Delta_{\text {wrinkling }}$ is the frequency model that relates the speed of the roller with the deflection in the form of

$$
\Delta_{\text {wrinkling }}=B \sin (w t),
$$

where $B$ is the parameter of amplitude for the displacement, $w$ is the number of waves and $t$ is the time of the function. Considering $t=l_{s} / r_{x}$ the equation takes the form

$$
\Delta_{\text {wrinkling }}=B \sin \left(w \frac{l_{s}}{r_{x}}\right)
$$

where $l_{S}$ is the length of the material sheet and the $r_{x}$ is the speed of the roller over the material.

In the Eq. (41), the definition of $B$ gives the error for the specific defect presented here. As a conclusion for the section, the deviation due to the panel for Eq. (18) takes the form of

$$
\begin{aligned}
\Delta_{\text {sheet }}= & -\left(\frac{F_{e}}{F}\right)^{2}\left[5-\left(3+\frac{F}{F_{e}}\right) \sqrt{\left.3-\frac{2 F}{F_{e}}\right] \frac{F l^{3}}{3 E I},}\right. \\
& +B \sin \left(w \frac{l_{s}}{r_{x}}\right)
\end{aligned}
$$

completing the model for deformation due to the panel.

\subsection{The Trajectory Compensation Model}

By integrating the deviation due to the robot and the deviation due to the panel into the Eq. (1), the complete model would take the form of

$$
\begin{aligned}
X_{n}^{W}= & X_{d}^{W} \\
& +K_{\theta}^{-1} J(\Theta) J^{T}(\Theta) F^{W} \\
& +R_{T}^{W}\left\{\hat{Z}\left\{-\left(\frac{F_{e}}{F}\right)^{2}\left[5-\left(3+\frac{F}{F_{e}}\right) \sqrt{3-\frac{2 F}{F_{e}}}\right] \frac{F^{T} l^{3}}{3 E I}\right\}\right\}, \\
& +B \sin \left(w \frac{l_{s}}{r_{x}}\right)
\end{aligned}
$$

where $R_{T}^{W}$ is the transformation matrix form the tool reference frame to the world reference frame and the $\hat{Z}$ indicates the force is acting only in that component.

\section{EXPERIMENTAL}

\subsection{The Stiffness/Compliance of the Robot}

In order to compute the joint stiffness matrix $k_{\theta}$ the joint stiffness values are model as functions of force and displacement.

The proposal of the experiment consists in evaluating two different positions with the same value for $Y$ and $Z$ axis and different for $X$ axis like the Fig. 8 shows, where the robot holds the roll hemming tool with a force sensor and two locations $P_{1}$ and $P_{2}$ are selected. In the two positions the intensity of the induced force in the $Z$ axis direction was changed according to the Table 2, the force was varied from 20 $\mathrm{N}$ to $120 \mathrm{~N}$ in different sub-steps.

The Fig. 9 shows an instrument to apply an external load on the tool, where the $Z$ in the reference

Table 2. Parameters of the experiment

\begin{tabular}{llc} 
Position [mm] & Run & Force $[\mathrm{N}]$ \\
\hline$x=660$ & 1 & 20 \\
\hline$y=0$ & 2 & 40 \\
\hline$z=-350$ & 3 & 60 \\
\hline & 4 & 80 \\
\hline & 5 & 100 \\
\hline \hline$x=425$ & 6 & 120 \\
\hline$y=0$ & 7 & 20 \\
\hline$z=-350$ & 8 & 40 \\
\hline & 9 & 60 \\
\hline & 10 & 80 \\
\hline & 11 & 100 \\
\hline & 12 & 120 \\
\hline
\end{tabular}




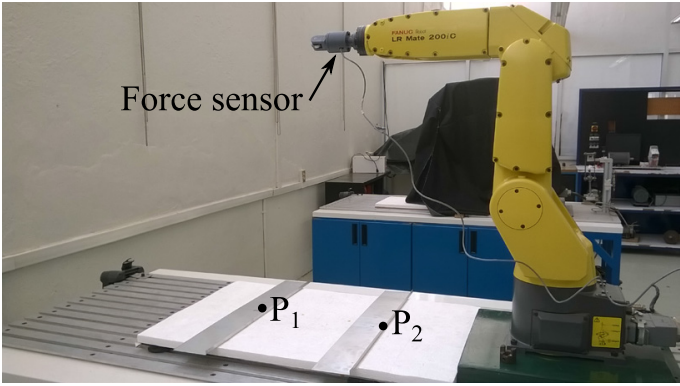

Fig. 8. The robot and two work space location for different stiffness performance

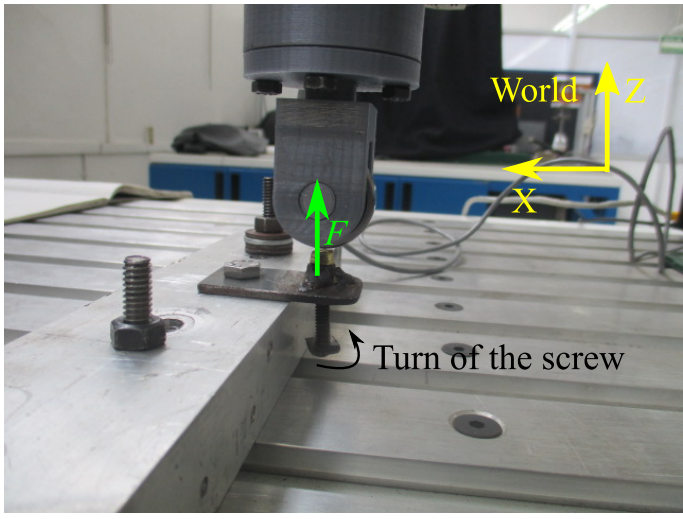

Fig. 9. An instrument to apply an external force incrementally

frame corresponds to the direction of the force, this instrument increments a specific force accordingly to the displacement obtained from the turn of the screw, the distance is controlled and the force was applied in the TCP $Z$ axis global direction.

\subsection{The Tests of Deformation of the Panel}

The experiment determines the process forces using a milling machine to avoid the compliance of the robot. One pre-hemming step at different low and high constant speeds was implemented. The Fig. 10(a) illustrates the tool end effector attached to the milling machine. The tool is at $45^{\circ}$ in order to apply the first pre-hemming step along the flange, the milling machine is considered as a rigid machine with infinite stiffness and the sheet is fixed and constrained in its movement, the force sensor in the tool that measures the signals during the tests is observed in the figure. The material for the panel sheet was aluminum 6000 series, the angle of the pre-hemming step was $45^{\circ}$, the length of the panel was $60 \mathrm{~mm}$, the force was measured in the $Z$ axis of the tool end effector at different constant speeds.

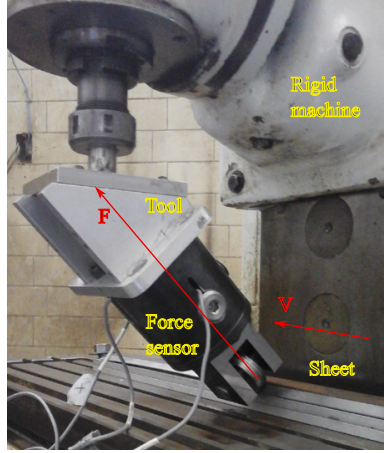

(a)

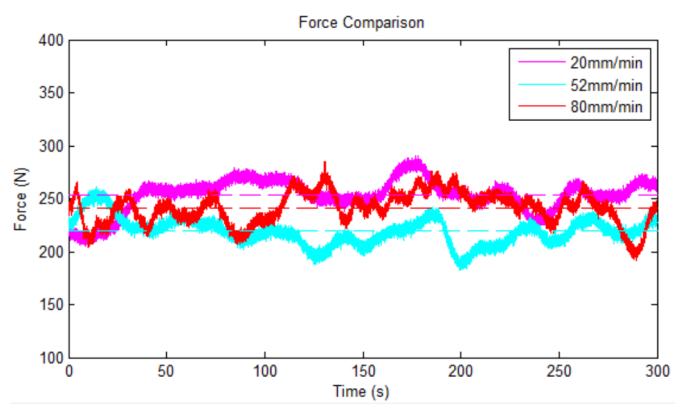

(b)

Fig. 10. The roll hemming tests; a) The experimental set up, b) The forces measured in the tests

The reported values of the forces were $200 \mathrm{~N}$ to $300 \mathrm{~N}$ which agrees well for a pre-hemming step. In the graph in Fig. 10(b), the force varies at different speeds due to material fluctuations. Three different data are observed, one for the speed at $20 \mathrm{~mm} / \mathrm{min}$, other at $52 \mathrm{~mm} / \mathrm{min}$ and the last graph at $80 \mathrm{~mm} / \mathrm{min}$, all these graphs were taken at the same pre-hemming step and different averages are observed, which means that the initial set up of the sheet was different for every one, also the graphs present different fluctuation patterns corresponding to the material deformation.

\subsection{The Roll Hemming Set Up}

The robot Fanuc 200IC with capacity of $200 \mathrm{~N}$ of payload in the tool was implemented for the experiment and the simulation, it is an industrial serial robot with 6 joints (6R). This robot is useful for tests at small scale since the reduced parameters allow it for easy and practical implementations.

The Fig. 11 shows the roll hemming tool in the upper part of the plot with the reference frame according to the tool frame and attached to the robot, it has a sensor to measure the force. In the bottom of the plot it is observed the frame attached to the metal 


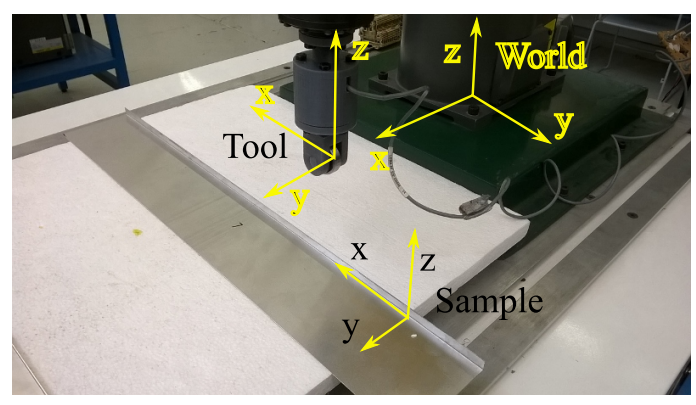

Fig. 11. The reference frames in the tool and the sample sheet

Table 3. The trajectory of the robot

\begin{tabular}{|c|c|}
\hline Run & Position [mm] \\
\hline & $Y=0 \quad Z=-326.319$ \\
\hline & $X$ \\
\hline 1 & 425 \\
\hline 2 & 451 \\
\hline 3 & 477 \\
\hline 4 & 503 \\
\hline 5 & 529 \\
\hline 6 & 555 \\
\hline 7 & 581 \\
\hline 8 & 607 \\
\hline 9 & 633 \\
\hline 10 & 660 \\
\hline
\end{tabular}

sheet and with the $X$ component coincident to the tool frame. The global frame that is attached to the base of the robot is also shown in the picture, according to this global reference frame the compensation strategy should work during the analysis of deviation.

The Table 3 shows the different location in the $X$ coordinate that were considered for the simulation, in this case the values of $Y$ and $Z$ coordinates remained constant and a total of ten points were obtained, for the simulation a force is applied in direction to the $Z$ global reference coordinate.

\section{RESULTS}

According to section 2.1. The graph of Fig. 12 plots the stiffness calculation giving positive and negative values, those negative values result from the direction of displacements and the Jacobian configuration; there is no value of $K_{6}$ displayed in the figure, for this experiment all the $K_{6}$ are infinite due to the force is acting in the $Z$ axis direction impeding its rotation. Also as we can observe from the figure the values of $K_{1}$ in the sixth run and $K_{2}$ in the second run overpass the graph scale, the two values are considered as atypical data. The values of all the runs, except the two atypical data, were averaged to result in the values of the Table

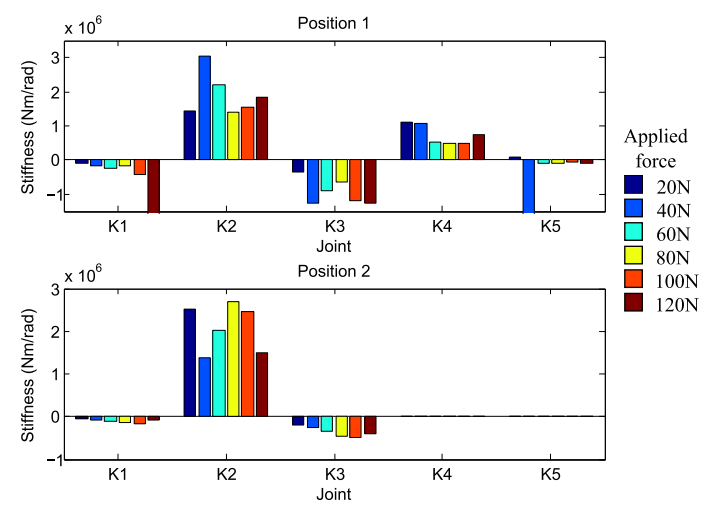

Fig. 12. The joint stiffness values computed for each joint

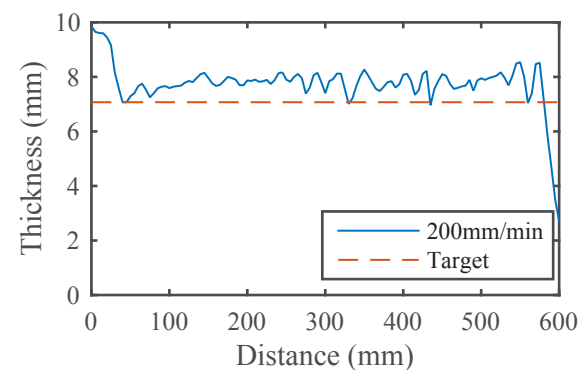

Fig. 13. The wrinkling defect at $200 \mathrm{~mm} / \mathrm{min}$

4. According to section 2.2. The Fig. 13 plots the graph of thickness and the distance along the sheet over the edge, the speed of the roll hemming was $200 \mathrm{~mm} / \mathrm{min}$ which represents a high speed for the process, in the figure it is observed that the wrinkling defect appear in high frequency and the error of the path and the target path is high. This means that the wrinkling defect has a direct relation with the speed of the process increasing the error and deviating the trajectory along the edge of the sheet.

The graph in Fig. 14 shows the result of the compensation strategy considering a constant force applied to the tool end effector. At the initial position of the tool the distance is positive and as the roller moves in $x$ direction the trajectory goes down until the value of the position in $z$ is negative, this may be because the compensations considering the deviation of the tool due to the low stiffness and the configuration of the robot. This graph shows that at the end of the trajectory the value of the trajectory could be under the desired position. The force applied is considered as constant and the value of the deviation is around the $4 \times 10^{-3} \mathrm{~mm}$ which is a low value in comparison with the location of the tool and the work-space. 
Table 4. The average of the stiffness values

\begin{tabular}{cc} 
Joint 1 & $\begin{array}{c}\text { Mean stiffness } \\
{[\mathrm{Nm} / \mathrm{rad}]}\end{array}$ \\
\hline \hline 1 & $-0.160 \times 10^{6}$ \\
\hline 2 & $2.001 \times 10^{6}$ \\
\hline 3 & $-0.651 \times 10^{6}$ \\
\hline 4 & $0.370 \times 10^{6}$ \\
\hline 5 & $-0.020 \times 10^{6}$ \\
\hline 6 & inf \\
\hline
\end{tabular}

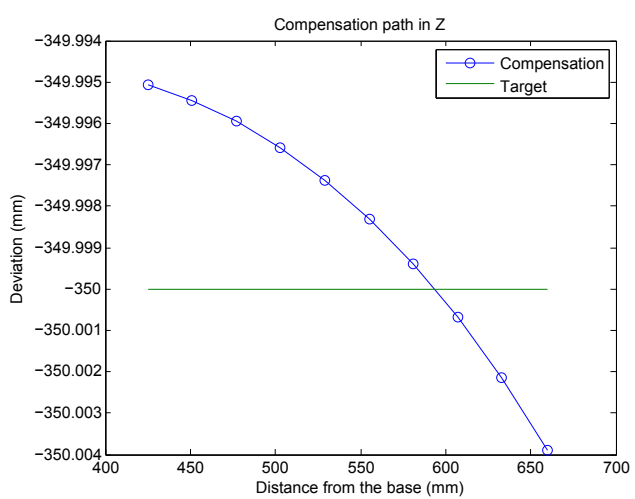

Fig. 14. Deviation in $Z$ constant force

The Fig. 15 shows the trajectory after the compensation is implemented considering a variable force applied in the robot, it is observed that as the roller moves over the sheet the trajectory goes to the negative values this is because the stiffness in that point is low, the difference with the graph shown before is that this compensation trajectory considers the wrinkling defect of the material since the values of the force were obtained from the experimental behavior of the panel.

The Fig. 16 shows the thickness of the tested samples as a function of the distance along the flange. In the figure, the reference is at $1 \mathrm{~mm}$ of thickness due to the flange thickness is $0.5 \mathrm{~mm}$ and the pre-hemming steps were $45^{\circ}$ and $0^{\circ}$. The trajectory without compensation appears slightly over the reference with a maximum thickness of $3.1 \mathrm{~mm}$ and the trajectory with the compensation appears closer to the reference with a maximum thickness of $2.8 \mathrm{~mm}$.

\section{DISCUSSIONS}

The range of the error for the compensation strategy is $1 \times 10^{-3} \mathrm{~mm}$, so the robot characteristics should meet this requirement. For example, the Fanuc 200IC accuracy for the TCP location is $1 \times 10^{-2} \mathrm{~mm}$. Which means that this robot finds out limitations to implement

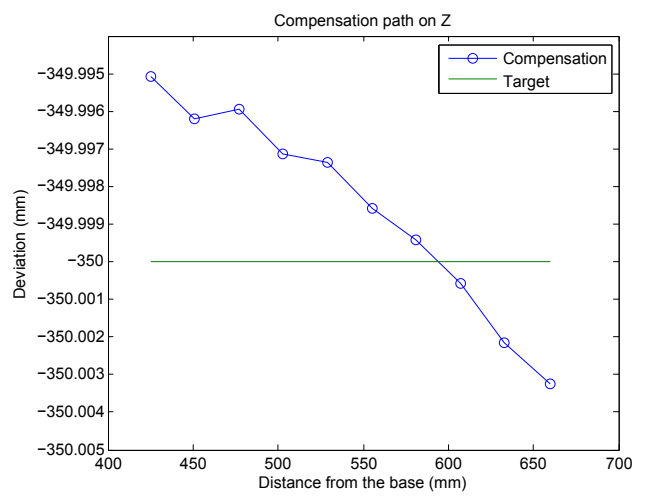

Fig. 15. Deviation in $Z$ with variable force

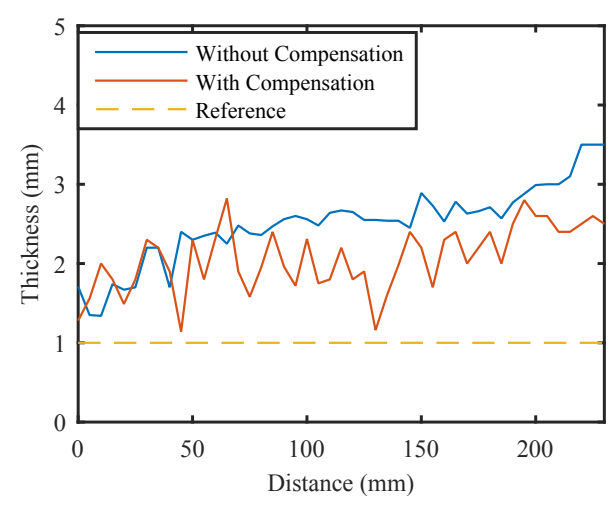

Fig. 16. The thickness of the sample sheets

this compensation strategy. Also, the accuracy of the previous process to the roll hemming will define the effectiveness of the compensation model. These graph results of Fig. 16 agree well compared to those of Posada et al. [7] where the translation errors were smaller than $0.1 \mathrm{~mm}$. Posada et al. implemented a sensor-based stiffness compensation model instead of a model-based. He discussed that the sensor-based proposal, which takes the real values, ensures better results than a model-based system, which predicts via simulation.

In contrast, the experimental results of Kamali et al. [8] revealed the proposed elasto-geometrical calibration approach of the TCP is able to reduce the maximum position error to $0.960 \mathrm{~mm}$. Such results agree well to the simulation results when the compensation method is implemented.

Hu et al. [9] developed a method to calculate the fracture limits of the material by developing several tests on the material fibers but a relation between the material properties and the robot parameters is not presented. LeMaout et al. [10] focused on the 
finite element analysis for pre-strain materials in roll hemming process. One of the focuses was to deal with the complex geometries of the panels. The authors of this paper want to encourage similar works looking for improvements to the material analysis and the FEM solutions by relating the robot parameters.

The works framed by Kohrt et al. [11] on posture optimization may be enriched by the results presented in this paper. The present paper may be cited by future works of roll hemming applications since the authors have been working on such topics [12].

\section{CONCLUSIONS}

This paper established the main problems of roll hemming process and an offline compensation strategy based on the variant force. The compensation proposal integrated a method to determine the deviation due to the robot and the deviation due to the panel. An improvement of $1 \mathrm{~mm}$ was observed from the results. The authors of this paper believe that if this model is enhanced and implemented in the industry, it may bring a complete solution for the roll hemming technology since the simulation with the experimental data showed good tendency in the correction of the trajectory during the process. Future works will consider: the previous internal strains of the material, the FEM simulation and a sensibility analysis of the variables.

\section{ACKNOWLEDGEMENTS}

The first author wishes to gratefully acknowledge Universidad Autónoma de Querétaro through grant scholarship of Conacyt (Consejo Nacional de Ciencia y Tecnología) for permitting his period of study at LaRM of Cassino University in the year 2016 within a double $\mathrm{PhD}$ degree program.

\section{REFERENCES}

[1] Guillo, M., Dubourg, L. (2016). Impact \& improvement of tool deviation in friction stir welding: Weld quality \& real-time compensation on an industrial robot. Robotics and ComputerIntegrated Manufacturing, vol. 39, p. 22-31, D0I:10.1016/j. rcim.2015.11.001.
[2] Thuillier, S., Le Maoût, N., Manach, P.Y., Debois, D. (2008). Numerical simulation of the roll hemming process. Journal of Materials Processing Technology, vol. 198, no. 1-3, p. 226233, D0l:10.1016/j.jmatprotec.2007.07.004.

[3] Zhang, H., Wang, J., Zhang, G., Gan, Z., Pan, Z., Cui, H., Zhu, Z. (2005). Machining with flexible manipulator: toward improving robotic machining performance. Proceedings of IEEE/ASME International Conference on Advanced Intelligent Mechatronics, p. 1127-1132, D0l:10.1109/ AIM.2005.1511161.

[4] Le Maoût, N., Manach, P.Y., Thuillier, S. (2012). Influence of prestrain on the numerical simulation of the roller hemming process. Journal of Materials Processing Technology, vol. 212, no. 2, p. 450-457. D0l:10.1016/j.jmatprotec.2011.10.008.

[5] Drossel, W.-G., Pfeifer, M., Findeisen, M., Rössinger, M., Eckert, A., Barth, D. (2014). The influence of the robot's stiffness on roller hemming processes. 45th International Symposium on Robotics, p. 531-538.

[6] Chakrabarty, J. (2006). Theory of Plasticity, 3rd Edition, Butterworth-Heinemann Elsevier, Oxford, .

[7] Diaz Posada, J.R., Schneider, U., Pidan, S., Geravand, M., Stelzer, P., Verl, A. (2016). High accurate robotic drilling with external sensor and compliance model-based compensation. Proceedings of IEEE International Conference on Robotics and Automation, p. 3901-3907. DOI:10.1109/ICRA.2016.7487579.

[8] Kamali, K., Joubair, A., Bonev, I.A., Bigras, P. (2016). Elasto-geometrical calibration of an industrial robot under multidirectional external loads using a laser tracker. Proceedings of IEEE International Conference on Robotics and Automation, p. 4320-4327, D0l:10.1109/ ICRA.2016.7487630.

[9] Hu, X., Zhao, Y., Huang, S., Li, S., Lin, Z. (2012). Numerical analysis of the roller hemming process. The International Journal of Advanced Manufacturing Technology, vol. 62, no. 5-8, p. 543-550, DOI:10.1007/s00170-011-3822-4.

[10] Le Maoût, N., Thuillier, S., Manach, P.Y. (2010). Classical and roll-hemming processes of pre-strained metallic sheets. Experimental Mechanics, vol. 50, no. 7, p. 1087-1097, D0l:10.1007/s11340-009-9297-7.

[11] Kohrt, C., Stamp, R., Pipe, A.G., Kiely, J., Schiedermeier, G. (2013). An online robot trajectory planning and programming support system for industrial use. Robotics and ComputerIntegrated Manufacturing, vol. 29, no. 1, p. 71-79, D0I:10.1016/j.rcim.2012.07.010.

[12] Esquivel, E., Carbone, G., Ceccarelli, M., Jáuregui, J. (2017). Requirements and constraints for a robotized roll hemming solution. RAAD 2016: Advances in Robot Design and Intelligent Control, Springer, Cham, p. 244-251, DOI:10.1007/978-3-319-49058-8_27. 